J. Sanabria, E. Rosas, L. VÁsquez

\title{
ON INVERSELY $\theta$-SEMI-OPEN AND INVERSELY $\theta$-SEMI-CLOSED FUNCTIONS
}

\begin{abstract}
J. Sanabria, E. Rosas, L. Vásquez. On inversely $\theta$-semi-open and inversely $\theta$-semi-closed functions, Mat. Stud. 53 (2020), 92-99.

In this paper, we introduce the concepts of inversely $\theta$-semi-open and inversely $\theta$-semi-closed functions and obtain their characterizations if it is possible in terms of $\theta$-closure and $\theta$-interior by using sets determined by the fibres of the function. Finally, we obtain its relationships with the strongly $\theta$-continuous.
\end{abstract}

1. Introduction and preliminaries. The notion of semi-open set in a topological space, which is one of the generalizations of open sets, plays an important role in several of the recent research in General Topology. This notion was originally given in 1963 by Levine [5], who demonstrated that the family of semi-open sets is closed under arbitrary unions and the family of interiors of semi-open sets coincides with the topology of space. Semi-open sets have been used to define and study new versions of interior, closure, separation axioms and continuity. In particular, in 2011, Noorie and Ahuja [11] introduced and characterized the notions of inversely semi-open and inversely semi-closed functions in terms of semi closure and semi interior by using sets determined by the fibres of the function, similar to the inversely open and inversely closed functions given in [9]. Following this line of research, in this paper we introduce the notions of inversely $\theta$-semi-open and inversely $\theta$-semi-closed functions and establish their characterizations in terms of $\theta$-closure and $\theta$-interior [12] by using sets determined by the fibres of the function. In addition, several corollaries are obtained and finally we obtain a sufficient condition for a function to be strongly $\theta$-continuous [6].

Throughout this paper, $X$ and $Y$ always mean topological spaces on which no separation axioms are assumed unless explicitly stated. If $A$ is a subset of $X$, then we denote the closure of $A$, the interior of $A$ and complement of $A$ by $\operatorname{cl}(A), \operatorname{int}(A)$ and $A^{C}$, respectively. A subset $A$ of $X$ is said to be semi-open [5] if there exists an open set $U$ in $X$ such that $U \subset A \subset \operatorname{cl}(U)$. This is equivalent to say that $A \subset \operatorname{cl}(\operatorname{int}(A))$. The complement of a semi-open set is called a semi-closed set. The semi-closure of $A$, denoted by $\operatorname{scl}(A)$, is defined by the intersection of all semi-closed sets containing $A$. The semi-interior of $A$, denoted by $\operatorname{sint}(A)$, is $\operatorname{defined}$ by the union of all semi-open sets contained in $A$. A point $x \in X$ is called a $\theta$-cluster point of $A$ if $\operatorname{cl}(U) \cap A \neq \varnothing$ for each open set $U$ containing $x$ (see [12]). The set of all $\theta$-cluster points of $A$ is called the $\theta$-closure of $A$ and is denoted by $\theta-\operatorname{cl}(A)$. A subset $A$ of $X$ is said to be $\theta$-closed if $A=\theta-\operatorname{cl}(A)$. The complement of a $\theta$-closed set is said to be a $\theta$-open set.

2020 Mathematics Subject Classification: 54C05, 54C08, 54C10.

Keywords: inversely semi-open function; $\theta$-open set; $\theta$-closed set; inversely $\theta$-semi-open function; inversely $\theta$-semi-open function; strongly $\theta$-continuous function.

doi:10.30970/ms.53.1.92-99

(C) J. Sanabria, E. Rosas, L. Vásquez, 2020 
Similarly, the $\theta$-interior of a set $A$ in $X$, written $\theta$-int $(A)$, consists of those points $x$ of $X$ such that for some open set $U$ containing $x, \operatorname{cl}(U) \subset A$. It is well known that a subset $A$ of $X$ is $\theta$-open if and only if $A=\theta$-int $(A)$. It follows from [12] that the collection of all $\theta$-open sets in a topological space $X$ forms a topology on $X$ which is denoted by $\tau_{\theta}$. Observe that $\theta$ - cl is not the closure of A with respect to $\tau_{\theta}$. A function $f: X \rightarrow Y$ is called semiopen [1] (resp. semi-closed [2], pre-semi-open [4]) if the image of every open (resp. closed, semi-open) subset of $X$ is semi-open (resp. semi-closed, semi-open) in $Y$. Also, a function $f: X \rightarrow Y$ is called irresolute [4] (resp. strongly $\theta$-continuous [6]) if the inverse image of every semi-open (resp. open) subset of $Y$ is semi-open (resp. $\theta$-open) in $X$, and $f$ is said to be a semi-homeomorphism [4] if $f$ is bijective, irresolute and pre-semi-open. According to [11], a function $f: X \rightarrow Y$ is called inversely semi-open (resp. inversely semi-closed) if for each subset $A$ of $X, \operatorname{sint}(f(A)) \subset f(\operatorname{int}(A))($ resp. $f(\operatorname{cl}(A)) \subset \operatorname{scl}(f(A)))$. Recall that for any sets $X$ and $Y$, a subset $E$ of $X$ will be called a saturated [8] subset of $X$ under a map $f: X \rightarrow Y$ if for some subset $B$ of $Y, E=f^{-1}(B)$, or equivalently, if $E=f^{-1}(f(E))$.

Notation 1. For any sets $X$ and $Y$, let $f: X \rightarrow Y$ be any function and $E$ any subset of $X$. Then we will use the following notation:

1. The fiber of an element $y \in Y$ is denoted by $f^{-1} y$ and is defined as $f^{-1} y=\{x \in$ $X: f(x)=y\}$.

2. $f^{\#}(E)=\left\{y \in Y: f^{-1} y \subset E\right\}$.

3. $E^{\#}=f^{-1}\left(f^{\#}(E)\right)$.

The following lemma will be useful in the sequel.

Lemma 1 ([10]). For any sets $X, Y$, let $f: X \rightarrow Y$ be any function and $E$ be any subset of $X$. Then:

1. $E^{\#}=\left\{f^{-1} y: y \in Y\right.$ and $\left.f^{-1} y \subset E\right\} \subset E$.

2. $f^{\#}\left(E^{C}\right)=[f(E)]^{C}, f^{\#}(E)=\left[f\left(E^{C}\right)\right]^{C}$ and $\left[f^{\#}\left(E^{C}\right)\right]^{C}=f(E)$.

3. $E$ is saturated if and only if $E=E^{\#}$.

4. $f$ is onto if and only if $f^{\#}(A)=f\left(A^{\#}\right)$ for each subset $A$ of $X$.

Theorem 1 ([4]). If $f: X \rightarrow Y$ is semi-homeomorphism, then $f(\operatorname{sint}(A))=\operatorname{sint}(f(A))$, for any subset $A$ of $X$.

Theorem 2 ([4]). If $f: X \rightarrow Y$ is semi-homeomorphism, then $f(\operatorname{scl}(A))=\operatorname{scl}(f(A))$, for any subset $A$ of $X$.

2. Inversely $\theta$-semi-open functions. In this section, the inversely $\theta$-semi-open and inversely $\theta$-semi-closed functions are characterized. Also, we give conditions under which such functions are strongly $\theta$-continuous.

Definition 1. A function $f: X \rightarrow Y$ is called inversely $\theta$-semi-open if for each subset $A$ of $X, \operatorname{sint}(f(A)) \subset f(\theta-\operatorname{int}(A))$.

Example 1. Let $X=\{a, b, c\}, \tau_{1}=\{\varnothing, X,\{a\},\{b\},\{a, b\},\{b, c\}\}, \tau_{2}=\{\varnothing, X,\{a\}\}$. A function $f:\left(X, \tau_{1}\right) \rightarrow\left(X, \tau_{2}\right)$ defined by

$$
f(x)=\left\{\begin{array}{lll}
c, & \text { if } & x \neq b \\
b, & \text { if } & x=b
\end{array}\right.
$$

is inversely $\theta$-semi-open. 
Every inversely $\theta$-semi-open function is inversely semi-open. The following example shows that an inversely semi-open function need not be inversely $\theta$-semi-open.

Example 2. Let $X=\{a, b, c\}, \tau_{1}=\{\varnothing, X,\{a\},\{b\},\{a, b\},\{b, c\}\}, \tau_{2}=\{\varnothing, X,\{a\},\{b\},\{a, b\}$, $\{a, c\}\}$. A function $f:\left(X, \tau_{1}\right) \rightarrow\left(X, \tau_{2}\right)$ defined by

$$
f(x)=\left\{\begin{array}{lll}
c, & \text { if } & x \neq b \\
b, & \text { if } & x=b
\end{array}\right.
$$

is inversely semi-open but is not inversely $\theta$-semi-open.

In the following result, we characterize the inversely $\theta$-semi-open functions.

Theorem 3. For any function $f: X \rightarrow Y$, the following statements are equivalent:

1. $f$ is inversely $\theta$-semi-open.

2. $f^{\#}(\theta-\operatorname{cl}(A)) \subset \operatorname{scl}\left(f^{\#}(A)\right)$ for each subset $A$ of $X$.

3. If $V$ is a semi-open subset of $Y$ and $V \subset f(X)$, then each set consisting of exactly one point and so at least one point from each fiber $f^{-1} y$, where $y \in V$, is $\theta$-open in $X$.

4. For any subset $A$ of $X, A$ is $\theta$-open in $X$ whenever $f(A)$ is semi-open in $Y$.

5. For any subset $A$ of $X, A$ is $\theta$-closed en $X$ whenever $f^{\#}(A)$ is semi-closed in $Y$.

Proof. (a) $\Leftrightarrow$ (b) Let $A$ be any subset of $X$ and $f$ be an inversely $\theta$-semi-open function. Put $B=A^{C}$. Then $\operatorname{sint}(f(B)) \subset f(\theta-\operatorname{int}(B))$. Taking complement and using Lemma 1, we obtain that $[f(\theta-\operatorname{int}(B))]^{C} \subset[\operatorname{sint}(f(B))]^{C}$ and

$$
\begin{gathered}
f^{\#}(\theta-\operatorname{cl}(A))=\left[f\left([\theta-\operatorname{cl}(A)]^{C}\right)\right]^{C}=\left[f\left(\theta-\operatorname{int}\left(A^{C}\right)\right)\right]^{C}=[f(\theta-\operatorname{int}(B))]^{C} \subset \\
\subset[\operatorname{sint}(f(B))]^{C}=\left[\operatorname{sint}\left(f\left(A^{C}\right)\right)\right]^{C}=\operatorname{scl}\left(\left[f\left(A^{C}\right)\right]^{C}\right)=\operatorname{scl}\left(f^{\#}(A)\right) .
\end{gathered}
$$

Conversely, for any $A \subset X$ we have

$$
\begin{aligned}
{[f(\theta-\operatorname{int}(A))]^{C} } & =\left[f\left(\theta-\operatorname{int}\left(\left[A^{C}\right]^{C}\right)\right)\right]^{C}=\left[f\left(\left[\theta-\operatorname{cl}\left(A^{C}\right)\right]^{C}\right)\right]^{C}=f^{\#}\left(\theta-\operatorname{cl}\left(A^{C}\right)\right) \subset \\
& \subset \operatorname{scl}\left(f^{\#}\left(A^{C}\right)\right)=\operatorname{scl}\left([f(A)]^{C}\right)=[\operatorname{sint}(f(A))]^{C} .
\end{aligned}
$$

Taking complement we conclude that $\operatorname{sint}(f(A)) \subset f(\theta-\operatorname{int}(A))$ and hence, $f$ is inversely $\theta$-semi-open.

(a) $\Rightarrow$ (c) Let $f$ be an inversely $\theta$-semi-open function and $V$ be any semi-open subset of $Y$ such that $V \subset f(X)$. Let $S$ any set consisting of exactly one point from each fiber $f^{-1} y, y \in V$. It is clear that $f(S)=V$. Suppose that $S$ is not a $\theta$-open set in $X$, then $\theta$ - $\operatorname{int}(S) \varsubsetneqq S$. Since $S$ consisting of exactly one point from each fiber $f^{-1} y, y \in V$, it follows that $f(\theta-\operatorname{int}(S)) \neq f(S)=V$. By (a), we have $\operatorname{sint}(V)=\operatorname{sint}(f(S)) \subset f(\theta-\operatorname{int}(S))$ and as $f(\theta-\operatorname{int}(S)) \neq V$, then we have $\operatorname{sint}(V) \varsubsetneqq V$, which contradicts the fact that $V$ is a semi-open subset of $Y$. Therefore, $S$ is $\theta$-open in $X$.

$(\mathrm{c}) \Rightarrow(\mathrm{d})$ Let $V=f(A)$ be a semi-open subset of $Y$. Consider the collection, $\left\{S_{y}\right\}_{y}$ where each $S_{y}=f^{-1} y \cap A, y \in V$. Since $A=\bigcup\left\{S_{y}: y \in V\right\}$ and each $S_{y}$ contains at least one point from each fiber $f^{-1} y, y \in V$, we conclude by (c) that $A$ is a $\theta$-open set in $X$.

$(\mathrm{d}) \Rightarrow(\mathrm{e})$ For any set $A$ in $X$, let $f^{\#}(A)$ be a semi-closed set in $Y$. By Lemma 1 , we have $f^{\#}(A)=\left[f\left(A^{C}\right)\right]^{C}$ is semi-closed and so $f\left(A^{C}\right)$ is semi-open in $Y$. Then by $(\mathrm{d}), A^{C}$ is $\theta$-open in $X$ and hence, $A$ is $\theta$-closed in $X$. 
$(\mathrm{e}) \Rightarrow(\mathrm{d})$ Suppose that $f(A)$ is a semi-open set in $Y$. By Lemma $1,[f(A)]^{C}=f^{\#}\left(A^{C}\right)$ is semi-closed in $Y$ and by (e) it follows that $A^{C}$ is $\theta$-closed in $X$. Thus, $A$ is $\theta$-open in $X$.

$(\mathrm{d}) \Rightarrow(\mathrm{a})$ Let $A$ be any subset of $X$. Since $\operatorname{sint}(f(A)) \subset f(A)$, there exists a subset $E$ of $A$ such that $f(E)=\operatorname{sint}(f(A))$. Since $f(E)$ is a semi-open subset of $Y$, then by $(\mathrm{d}), E$ is a $\theta$-open set in $X$. Thus, $E \subset \theta-\operatorname{int}(A)$ and $f(E) \subset f(\theta-\operatorname{int}(A))$. Therefore, $\operatorname{sint}(f(A)) \subset f(\theta-\operatorname{int}(A))$ and so $f$ is inversely $\theta$-semi-open.

The following corollary gives the necessary condition under which an inversely $\theta$-semiopen function is strongly $\theta$-continuous.

Corollary 1. Let $f: X \rightarrow Y$ be inversely $\theta$-semi-open function. Then $f$ is strongly $\theta$-continuous if $f(X)$ is semi-open in $Y$.

Proof. Suppose that $f: X \rightarrow Y$ is an inversely $\theta$-semi-open function and let $G$ be any open set in $Y$. By [3, Theorem 1.9], $f\left(f^{-1}(G)\right)=G \cap f(X)$ is a semi-open set in $Y$ and by Theorem $3(\mathrm{~d}), f^{-1}(G)$ is a $\theta$-open set in $X$. Thus, $f$ is a strongly $\theta$-continuous function.

The following example shows that a strongly $\theta$-continuous injective function need not be inversely $\theta$-semi-open.

Example 3. Let $X=\{a, b, c\}, \tau_{1}=\{\varnothing, X,\{a\},\{b\},\{a, b\},\{b, c\}\}, \tau_{2}=\{\varnothing, X,\{a\}\}$. The function $f:\left(X, \tau_{1}\right) \rightarrow\left(X, \tau_{2}\right)$ defined by

$$
f(x)=\left\{\begin{array}{lll}
a, & \text { if } & x=a \\
b, & \text { if } & x=c \\
c, & \text { if } & x=b
\end{array}\right.
$$

is strongly $\theta$-continuous injective function but is not inversely $\theta$-semi-open.

Corollary 2. If $f: X \rightarrow Y$ is onto and inversely $\theta$-semi-open, then $f$ is strongly $\theta$-continuous.

Proof. This is an immediate consequence of Corollary 1.

Corollary 3. Let $f: X \rightarrow Y$ be onto and inversely $\theta$-semi-open, where each fiber in $X$ contains at least two points. Then $X$ is $T_{2}$.

Proof. Suppose that $f: X \rightarrow Y$ is an onto and inversely $\theta$-semi-open function such that each fiber in $X$ contains at least two points. Then $f\left(\{x\}^{C}\right)=Y$ for each $x \in X$ and since $f\left(\{x\}^{C}\right)=Y$ is a semi-open set in $Y$, by Theorem 3 (c), we have $\{x\}^{C}$ is a $\theta$-open set in $X$. Thus, for each $x \in X$, the singleton $\{x\}$ is $\theta$-closed in $X$, which shows $\left(X, \tau_{\theta}\right)$ is $T_{1}$, or equivalently by [7, Theorem 3], $X$ is $T_{2}$.

Corollary 4. Let $f: X \rightarrow Y$ be a constant function $f(x)=b$ for all $x \in X$ and for some $b \in Y$. Then:

1. If $f$ is an inversely $\theta$-semi-open and $\{b\}$ is semi-open in $Y$, then $X$ has the discrete topology.

2. If $\{b\}$ is not semi-open in $Y$, then $f$ is an inversely $\theta$-semi-open.

Proof. (a) Since each inversely $\theta$-semi-open function is inversely semi-open, this follows from Corollary 2.15 of [11].

(b) If $\{b\}$ is not semi-open in $Y$, then $\operatorname{sint}(f(A))=\operatorname{sint}(\{b\})=\varnothing \subset f(\theta-\operatorname{int}(A))$ for each subset $A$ of $X$. Hence, $f$ is inversely $\theta$-semi-open. 
Corollary 5. If $f: X \rightarrow Y$ is inversely $\theta$-semi-open and semi-homeomorphism, then $f(\operatorname{sint}(A))=f(\theta-\operatorname{int}(A))$ for each subset $A$ of $X$.

Proof. Let $A$ be any subset of $X$. Since $f$ is inversely $\theta$-semi-open, $\operatorname{sint}(f(A)) \subset f(\theta-\operatorname{int}(A))$ and as $f$ is a semi-homeomorphism, then by Theorem 1 , we have $f(\operatorname{sint}(A))=\operatorname{sint}(f(A)) \subset$ $f(\theta$-int $(A))$. Now, since inclusion $\theta-\operatorname{int}(A) \subset \operatorname{sint}(A)$ is always true, it follows that $f(\theta-\operatorname{int}(A))$ $\subset f(\operatorname{sint}(A))$. Therefore, $f(\operatorname{sint}(A))=f(\theta$-int $(A))$.

The following corollary shows that the image of saturated $\theta$-closed subsets under an inversely $\theta$-semi-open surjections is semi-closed.

Corollary 6. Let $f: X \rightarrow Y$ inversely $\theta$-semi-open and onto. Then for any saturated subset $A$ of $X, f(A)$ is semi-closed implies that $A$ is $\theta$-closed in $X$.

Proof. Suppose that $A$ is a saturated subset of $X$. By Lemma 1(c), we have $A=A^{\#}$. Since $f$ is onto, then we apply Lemma $1(\mathrm{~d})$ and get that $f(A)=f\left(A^{\#}\right)=f^{\#}(A)$ and as $f(A)$ is semi-closed, then by Theorem 3 (e), it follows that $A$ is $\theta$-closed in $X$.

3. Inversely $\theta$-semi-closed functions. In this section, we define and study the inversely $\theta$-semi-closed functions. Also, we show that such functions are strongly $\theta$-continuous.

Definition 2. A function $f: X \rightarrow Y$ is called inversely $\theta$-semi-closed if for each subset $A$ of $X, f(\theta-\operatorname{cl}(A)) \subset \operatorname{scl}(f(A))$.

Example 4. Consider $X=\{a, b, c\}, \tau_{1}=\{\varnothing, X,\{a\},\{c\},\{a, c\},\{b, c\}\}, \tau_{2}=\{\varnothing, X,\{b, c\}\}$. Define a function $f:\left(X, \tau_{1}\right) \rightarrow\left(X, \tau_{2}\right)$ by

$$
f(x)=\left\{\begin{array}{lll}
c, & \text { if } & x \neq b \\
b, & \text { if } & x=b .
\end{array}\right.
$$

It is easy to see that $f$ is an inversely $\theta$-semi-closed function.

Every inversely $\theta$-semi-closed function is inversely semi-closed. The following example shows that an inversely semi-closed function need not be inversely $\theta$-semi-closed.

Example 5. Let $X=\{a, b\}, Y=\{0,1\}, \tau_{1}=\{\varnothing, X,\{a\}\}$ and $\tau_{2}=\{\varnothing, Y,\{1\}\}$. Define function $f:\left(X, \tau_{1}\right) \rightarrow\left(Y, \tau_{2}\right)$ by $f(a)=1, f(b)=0$. It is easy to see that $f$ is inversely semi-closed but is not inversely $\theta$-semi-closed.

At this point, we have a question. It is possible to characterize inversely $\theta$-semi-closed functions in the same form as we did a characterization of inversely $\theta$-semi-open in Theorem 3 . The answer is in the negative, as we can see in the following theorem.

Theorem 4. For a function $f: X \rightarrow Y$, the following implications:

1. $(\mathrm{a}) \Leftrightarrow(\mathrm{b})$;

2. $(\mathrm{d}) \Leftrightarrow(\mathrm{e})$;

3. (a) $\Rightarrow(\mathrm{c})$;

4. $(\mathrm{a}) \nRightarrow(\mathrm{d})$.

hold: 
1. $f$ is inversely $\theta$-semi-closed.

2. $\operatorname{sint}\left(f^{\#}(A)\right) \subset f^{\#}(\theta-\operatorname{int}(A))$ for each subset $A$ of $X$.

3. If $G$ is a semi-closed subset of $Y$ and $G \subset f(X)$, then each set consisting of exactly one point and so at least one point from each fiber $f^{-1} y$, where $y \in G$, is $\theta$-closed in $X$.

4. For any subset $A$ of $X, A$ is $\theta$-closed in $X$ whenever $f(A)$ is semi-closed in $Y$.

5. For any subset $A$ of $X, A$ is $\theta$-open in $X$ whenever $f^{\#}(A)$ is semi-open in $Y$.

Proof. (1). Let $A$ be any subset of $X$ and suppose that $f$ is an inversely $\theta$-semi-closed function. Put $B=A^{C}$. Then $f(\theta-\operatorname{cl}(B)) \subset \operatorname{scl}(f(B))$. Taking complement and using Lemma 1, we have $[\operatorname{scl}(f(B))]^{C} \subset[f(\theta-\operatorname{cl}(B))]^{C}$ and

$$
\begin{gathered}
\operatorname{sint}\left(f^{\#}(A)\right)=\operatorname{sint}\left(\left[f\left(A^{C}\right)\right]^{C}\right)=\left[\operatorname{scl}\left(f\left(A^{C}\right)\right)\right]^{C}=[\operatorname{scl}(f(B))]^{C} \subset \\
\subset[f(\theta-\operatorname{cl}(B))]^{C}=\left[f\left(\theta-\operatorname{cl}\left(A^{C}\right)\right)\right]^{C}=\left[f\left([\theta-\operatorname{int}(A)]^{C}\right)\right]^{C}=f^{\#}(\theta-\operatorname{int}(A)) .
\end{gathered}
$$

Conversely, for any $A \subset X$ we have

$$
\begin{gathered}
{[\operatorname{scl}(f(A))]^{C}=\operatorname{sint}\left([f(A)]^{C}\right)=\operatorname{sint}\left(f^{\#}\left(A^{C}\right)\right)=f^{\#}\left(\theta-\operatorname{int}\left(A^{C}\right)\right) \subset} \\
\subset\left[f\left(\left[\theta-\operatorname{int}\left(A^{C}\right)\right]^{C}\right)\right]^{C}=\left[f\left(\theta-\operatorname{cl}\left(\left[A^{C}\right]^{C}\right)\right)\right]^{C}=[f(\theta-\operatorname{cl}(A))]^{C} .
\end{gathered}
$$

Taking complement we conclude that $f(\theta-\operatorname{cl}(A)) \subset \operatorname{scl}(f(A))$ and therefore, $f$ is inversely $\theta$-semi-closed.

(2). For any subset $A$ of $X$, let $f^{\#}(A)$ be a semi-open set in $Y$. By Lemma 1 , we have $f^{\#}(A)=\left[f\left(A^{C}\right)\right]^{C}$ is semi-open and so $f\left(A^{C}\right)$ is semi-closed in $Y$. Then by (d), $A^{C}$ is $\theta$-closed in $X$ and so, $A$ is $\theta$-open in $X$.

Conversely, suppose that $f(A)$ is a semi-closed in $Y$. By Lemma $1,[f(A)]^{C}=f^{\#}\left(A^{C}\right)$ is semi-open in $Y$ and by (e) it follows that $A^{C}$ is $\theta$-open in $X$. Hence, $A$ is $\theta$-closed in $X$.

(3). Let $f$ be an inversely $\theta$-semi-closed function and $G$ be any semi-closed subset of $Y$ such that $G \subset f(X)$. Let $H$ any set consisting of exactly one point from each fiber $f^{-1} y$, $y \in G$. It is clear that $f(H)=G$. Assume that $H$ is not a $\theta$-closed set in $X$, then $H \subset \theta-\operatorname{cl}(H)$ but $\theta-\operatorname{cl}(H) \neq H$ and so $G=f(H) \subset f(\theta-\operatorname{cl}(H))$ but $f(\theta-\operatorname{cl}(H)) \neq G$. By (a), we have $f(\theta-\operatorname{cl}(H)) \subset \operatorname{scl}(f(H))=\operatorname{scl}(G)$ and since $f(\theta-\operatorname{cl}(H)) \neq G$, it follows that $G \subset \operatorname{scl}(G)$ but $\operatorname{scl}(G) \neq G$, which contradicts the fact that $G$ is a semi-closed subset of $Y$. Therefore, $G$ is $\theta$-closed in $X$.

(4). See the following example.

Example 6. In Example 4, it is easy to see that the function $f$ is inversely $\theta$-semi-closed but it does not satisfies the condition (d) of Theorem 4, that is, for any subset $A$ of $X, A$ is $\theta$-closed in $X$ whenever $f(A)$ is semi-closed in $Y$.

At this point is necessary to do the following comment related with the article [11], specifically the results given in [11, Theorem 2.13]. The implication $(\mathrm{a}) \Rightarrow(\mathrm{d})$ is false, as we show in the following example.

Example 7. Consider $X=\{a, b, c\}, \tau_{1}=\{\varnothing, X,\{a\},\{b\},\{a, b\},\{a, c\}\}, \tau_{2}=\{\varnothing, X,\{a\}\}$. Define a function $f:\left(X, \tau_{1}\right) \rightarrow\left(X, \tau_{2}\right)$ by

$$
f(x)=\left\{\begin{array}{lll}
c, & \text { if } & x \neq b \\
b, & \text { if } & x=b .
\end{array}\right.
$$


It is easy to see that the function $f$ is inversely semi-closed but it does not satisfies the condition (d) of [11, Theorem 2.13] that is, for any subset $A$ of $X, A$ is closed in $X$ whenever $f(A)$ is semi-closed in $Y$.

Continuing with the article [11], we have noticed that the proof of [11, Corollary 2.14] is incorrect, because the authors used the equivalence of [11, Theorem 2.13]. In the following result we give an improved version of [11, Corollary 2.14], where the condition that the function $f$ is onto is not necessary.

Corollary 7. If $f: X \rightarrow Y$ is inversely semi-closed, then $f$ is continuous.

Proof. Let $G$ be any closed set in $Y$. Put $A=f^{-1}(G)$. Since $f$ is inversely semi-closed, $f(\operatorname{cl}(A)) \subset \operatorname{scl}(f(A))$ and so $f\left(\operatorname{cl}\left(f^{-1}(G)\right)\right) \subset \operatorname{scl}\left(f\left(f^{-1}(G)\right)\right) \subset \operatorname{scl}(G)$. Since $G$ is closed in $Y$, it is semi-closed in $Y$ and hence $\operatorname{scl}(G)=G$. Thus, $f\left(\operatorname{cl}\left(f^{-1}(G)\right)\right) \subset G$, which implies that $\operatorname{cl}\left(f^{-1}(G)\right) \subset f^{-1}(G)$ and so $\operatorname{cl}\left(f^{-1}(G)\right)=f^{-1}(G)$. Therefore, $f$ is continuous.

The following corollary shows that every inversely $\theta$-semi-closed function is strongly $\theta$-continuous.

Corollary 8. If $f: X \rightarrow Y$ is inversely $\theta$-semi-closed, then $f$ is strongly $\theta$-continuous.

Proof. Let $G$ be any closed set in $Y$. Put $A=f^{-1}(G)$. Since $f$ is inversely $\theta$-semi-closed, $f(\theta-\operatorname{cl}(A)) \subset \operatorname{scl}(f(A))$, that is $f\left(\theta-\operatorname{cl}\left(f^{-1}(G)\right)\right) \subset \operatorname{scl}\left(f\left(f^{-1}(G)\right)\right)$. But $\operatorname{scl}\left(f\left(f^{-1}(G)\right)\right) \subset$ $\operatorname{scl}(G)=G$, because $G$ is semi-closed in $Y$. Then, $f\left(\theta-\operatorname{cl}\left(f^{-1}(G)\right)\right) \subset G$ and hence

$$
\left.\theta-\operatorname{cl}\left(f^{-1}(G)\right)\right) \subset f^{-1}(G)
$$

Thus, $\left.\theta-\operatorname{cl}\left(f^{-1}(G)\right)\right)=f^{-1}(G)$ and $A=f^{-1}(G)$ is $\theta$-closed in $X$. This shows that $f$ is strongly $\theta$-continuous.

The following example shows a function $f$ that is strongly $\theta$-continuous but is not inversely $\theta$-semi-closed.

Example 8. Consider the function $f$ given in Example 3. Then, $f$ is strongly $\theta$-continuous. Now, if $A=\{c\}$, we have $f(\theta-\operatorname{cl}(A))=\{b, c\}$ and $\operatorname{scl}(f(A))=\{b\}$. It follows that $f$ is not inversely $\theta$-semi-closed.

Corollary 9. If $f: X \rightarrow Y$ is an inversely $\theta$-semi-closed and onto function, then each singleton in the fiber of a semi-closed singleton of $Y$ is $\theta$-closed in $X$.

Corollary 10. If $f: X \rightarrow Y$ is inversely $\theta$-semi-closed and semi-homeomorphism, then $f(\operatorname{scl}(A))=f(\theta-\operatorname{cl}(A))$ for any subset $A$ of $X$.

Proof. Let $A$ be any subset of $X$. Since $f$ is inversely $\theta$-semi-closed, then $f(\theta-\operatorname{cl}(A))$ $\subset \operatorname{scl}(f(A))$ and as $f$ is a semi-homeomorphism, then by Theorem 2, we have $f(\theta-\operatorname{cl}(A)) \subset$ $\operatorname{scl}(f(A))=f(\operatorname{scl}(A))$, obtaining $f(\theta-\operatorname{cl}(A)) \subset f(\operatorname{scl}(A))$. Now, since inclusion $\operatorname{scl}(A) \subset$ $\theta-\operatorname{cl}(A)$ is always true, it follows that $f(\operatorname{scl}(A)) \subset f(\theta-\operatorname{cl}(A))$. Hence, $f(\operatorname{scl}(A))=f(\theta-\operatorname{cl}(A))$. 


\section{REFERENCES}

1. Biswas N., On some mappings in topological spaces, Bull. Calcutta Math. Soc., 61 (1969), 127-135.

2. Biswas N., On characterizations of semi-continuous functions, Atti Accad. Naz. Lincei Rend. Cl. Sci. Fis. Mat. Natur., 48 (1970), 399-402.

3. Crossley S.G., Hildebrand S.K., Semi-closure, Texas J. Sci., 22 (1971), 99-112.

4. Crossley S.G., Hildebrand S.K., Semi-topological properties, Fund. Math., 74 (1972), 233-254.

5. Levine N., Semi-open sets and semi-continuity in topological spaces, Amer. Math. Monthly, 70 (1963), $36-41$.

6. Long P.E., Herrington L.L., Strongly $\theta$-continuous functions, J. Korean Math. Soc., 18 (1981), 21-28.

7. Mršević M., Andrijević D., $\theta$-connectedness and $\theta$-closure spaces, Topology Appl., 123 (2002), 157-166.

8. Noorie N.S., Bala R., Some characterizations of open, closed and continuous mappings, Internat. J. Math. \& Math. Sci. (2008), Article ID527106, 5 p.

9. Noorie N.S., Inversely open and inversely closed maps, Arya Bhatta J. Math. Inform., 3 (2011), №2.

10. Noorie N.S., Ahuja. J., Some characterizations of semi-open, semi-closed and semi-continuous mappings, Arya Bhatta J. Math. Inform., bf5 (2013), №2.

11. Noorie N.S., Ahuja J., Inversely semi-open and inversely semi-closed maps, J. Adv. Stud. Topol., 5 (2014), №3, 10-15.

12. Veličko N.V., H-closed topological spaces, Trans. Amer. Math. Soc., 78 (1968), 103-118.

Universidad de Sucre, Facultad de Educación y Ciencias

Departamento de Matemáticas, Sincelejo, Colombia

jesanabri@gmail.com

Universidad de la Costa, Departamento de Ciencias Naturales y Exactas, Barranquilla

Colombia \& Departamento en Matemáticas, Universidad de Oriente, Cumaná, Venezuela

ennisrafael@gmail.com

Instituto Superior de Formaciyn Docente Salomй Ureca-ISFODOSU, Recinto Emilio Prud'Homme

Los Pepines, Santiago de los Caballeros, Repablica Dominicana

luis.vasquez@isfodosu.edu.do 